\title{
Intermédialités
}

Histoire et théorie des arts, des lettres et des techniques

Intermediality

History and Theory of the Arts, Literature and Technologies

\section{Le martyre de sainte Sébastienne}

Retour sur une vision de Victor Burgin

\section{Vincent Bruyère}

Numéro 17, printemps 2011

reproduire

reproducing

URI : https://id.erudit.org/iderudit/1005752ar

DOI : https://doi.org/10.7202/1005752ar

Aller au sommaire du numéro

Éditeur(s)

Revue intermédialités (Presses de l’Université de Montréal)

ISSN

1705-8546 (imprimé)

1920-3136 (numérique)

Découvrir la revue

Citer cet article

Bruyère, V. (2011). Le martyre de sainte Sébastienne : retour sur une vision de Victor Burgin. Intermédialités / Intermediality, (17), 119-130.

https://doi.org/10.7202/1005752ar
Résumé de l'article

Cet article se propose de reproduire l'expérience hagiographique de Victor Burgin devant un autoportrait de Helmut Newton à partir d'interventions critiques de la part de Laura Mulvey, Michel de Certeau, Daniel Arasse et Georges Didi-Huberman. Au centre de cette expérience se situe la figure insaisissable de sainte Sébastienne ainsi que l'amorce de la question de sa différence en tant qu'événement à la fois visuel et de genre dans le discours de l'histoire sur l'image (d'art). Poursuivant le spectre de Sébastienne à travers ses diverses incarnations chez Helmut Newton, Louise Bourgeois et Antonello de Messine, l'objectif est de questionner l'opérativité critique du « re- » qui conditionne à la fois un retour à l'image et le retour du regard dans l'image. 


\title{
Le martyre de sainte Sébastienne
}

\section{Retour sur une vision de Victor Burgin}

\author{
VINCENT BRUYĖRE
}

Hagiography's desire to make visible
always edges up against the vision of desire.

I.

La sainte martyre dont il sera question n'existe pas vraiment. Son martyre n'est que le reflet d'une véritable icône de Helmut Newton, Self-Portrait with Wife June and Model (1981), dans l'imagination du photographe et critique Victor Burgin: «I think and the image of the woman in Newton's mirror returns to me as a sainte Sébastienne, waiting to receive time's arrows ${ }^{2}$. Telle est la réalité hagiographique qui est conférée à sainte Sébastienne par le texte «Newton’s Gravity». La sainteté de la pin-up de Newton s'articule dans les mêmes termes et avec les mêmes armes que le punctum barthésien : "Ce n’est pas moi qui vais le chercher, [...] c'est lui qui part de la scène, comme une flèche, et vient me percer $^{3}$. » Devant une image de sainteté qui n'en est pas une, au seuil même de l'image et du récit, au seuil du discours sur l'image qui prétend donner à voir, mieux voir, ou voir autrement, il ne sera dès lors pas seulement question de cette insistance, c'est-à-dire du retour sur l'image et du retour du regard dans l'image, mais également de ce qui est en jeu dans l'opérativité du préfixe re- (revenir,

1. Cary Howie, Claustrophilia. The Erotics of Enclosure in Medieval Literature, New York, Palgrave, 2007, p. $3^{8 .}$

2. Victor Burgin, «Newton's Gravity», dans In/Different Spaces. Places and Memory in Visual Culture, Berkeley, University of California Press, 1996, p. 91. Pour la photographie en question, voir Helmut Newton, Self-portrait with Wife June and Model, Paris, Vogue Studio, 1981. Reproduction en ligne: www.theartreserve.com/self-portrait (dernière consultation le 16 juin 2011).

3. Roland Barthes, La chambre claire. Note sur la photographie, Paris, Gallimard, coll. «Cahiers du Cinéma», 1980, p. 48-49. 
relire, reprendre, réviser, récompenser, restaurer et reproduire) signalant cette insistance. Réciproquement et relativement à cette dynamique, il sera question de la qualification du discours critique, de cette critique en particulier qui depuis un certain temps - et d'une historicité justement marquée par ce re- dans une problématique de l'instauration d'une discursivité - prête l'oreille aux heurts de la langue et du corps, aux ratés du symbolique, qui est devenue experte des phénomènes d'interruption, d'altération, d'intrusion, de déchirure, de mutilations et d'abjections en tout genre, et qui, ce faisant, signale la venue de l'autre. Il sera enfin question du récit de cette invention d'une alternative et de son historicité: « $[\mathrm{O}] \mathrm{n}$ ne fait pas venir l'autre, on le laisse venir en se préparant à sa venue. Le venir de l'autre ou son revenir, c'est la seule survenue possible, mais elle ne s'invente pas, même s'il faut la plus géniale inventivité qui soit pour se préparer à l'accueillir. ${ }^{4}$ » Ce phénomène de réciprocité reste l'énigme même que cet article s'efforce de poser et d'articuler sous forme de récit.

L'étude de l'autoportrait de Newton dans Perverse Space est un détour qui permet à Burgin de revenir sur le texte fondateur de Laura Mulvey «Visual Pleasure and Narrative Cinema ${ }^{5}$. Le propos de Burgin vise moins l'argumentation de Mulvey en tant que telle, que sa réduction et sa paradoxale fétichisation par un certain discours critique sur la culture visuelle. Il convient de rappeler que Mulvey prend pour point de départ la fascination qu'exerce le cinéma, le dispositif cinématographique constituant selon elle la prothèse d'un appareil social, linguistique et psychologique de subjectivation qu'il appartient à la psychanalyse à la fois de décrire et de désamorcer:

It is said that analysing pleasure, or beauty, destroys it. That is the intention of this article. The satisfaction and reinforcement of the ego that represent the high point of film history hitherto must be attacked. Not in favour of a reconstructed new pleasure, which cannot exist in the abstract, nor of intellectualized unpleasure, but to make way for a total negation of the ease and plenitude of the narrative fiction film ${ }^{6}$.

Mulvey poursuit en qualifiant la nature de l'événement critique qu'elle précipite dans le champ du visible: «The alternative is the drill that comes from leaving the past behind without rejecting it, transcending outworn or oppressive forms, or daring to break with normal pleasurable expectations in order to

4. Jacques Derrida, «Psyché. Invention de l'autre», dans Psyché. Inventions de l'autre, Paris, Galilée, 1998, p. 60.

5. Burgin, 1996, p. 57-76.

6. Laura Mulvey, «Visual Pleasure and Narrative Cinema», Screen, vol. 16, n 3 , 1975 , p. 8. 
conceive a new language of pleasure 7 . Il en va, dans ce frisson iconoclaste et dialectique, de la corrélation entre un désir d'altérer l'image et la définition d'un seuil du visible. Je tenterai de reproduire dans ce qui suit l'expérience hagiographique de Burgin afin de faire apparaître et de situer dans sa vision cette réciprocité critique.

\section{II.}

Dans Self-Portrait with Wife June and Model, Sébastienne se tient dans une composition spéculaire complexe d'ouvertures et de retraits du visible: une femme sculpturale pose nue - comme nudité - face à un miroir. Elle nous tourne le dos, tandis que nous voyons sa face cachée dans un reflet où figure également le photographe Helmut Newton. À hauteur du miroir, mais en dehors de son cadre, légèrement en retrait, June est assise. Derrière elle, le studio s'ouvre sur la rue. Entre l'objectif photographique et le tain du miroir, le spectacle du visible libère son volume, tout comme dans la composition des Ménines de Vélasquez, dont l'analyse sert d'ouverture à Les mots et les choses (1966). Le photographe opère, enveloppé dans un imperméable, la tête recroquevillée entre ses épaules, masqué par l'appareil photographique qui, dès lors, vaut comme la prothèse exorbitée de son œil. Son appareil fixe un point invisible, en réalité lui-même, en retrait dans l'ombre du dos du modèle, mais que nous les spectateurs nous pouvons aisément assigner puisque ce point c'est nous-mêmes, pris en flagrant délit. Le miroir creuse l'espace du studio répétant en cela l'ouverture de la porte qui théâtralise l'effraction du monde dans l'image. "Is the similarity of this image [Self-Portrait with Wife June and Model] to the Las Meninas of Vélasquez also due to chance $^{8}$ ? Hasard ou pas, la lecture de Foucault hante l'imaginaire critique, et c'est d'abord contre cette «foucaldianisation» du regard que s'inscrit Burgin explorant une géométrie non euclidienne du désir dans le champ de la vision, à la recherche d'un autre partage du lisible et du visible?.

Dans «Les suivantes », une représentation de la représentation s'organise autour de la présence du miroir trouble dans lequel se reflète une figuration du regard souverain tandis que s'efface l'inscription de son corps dans l'espace de la représentation. Parce qu'il se tient en marge du visible, parce que la composition lui assigne une position de retrait, et enfin parce qu'invisible, le

\section{Ibid.}

8. Burgin, 1996, p. 285. Voir aussi Michel Foucault, «Les suivantes», dans Les mots et les choses, Paris, Gallimard, coll. «Bibliothèque des sciences humaines», 1966.

9. Burgin, 1996, p. 66-67. 
souverain conditionne l'intégrité de la vision et du champ du visible. Il accomplit l'image comme image. Rien de semblable cependant dans l'image de Newton. L'évidence de la souveraineté du regard est précisément mise en question par le miroir. Sa présence troublante marque le seuil optique de ce que Burgin appelle «l'espace pervers» de cette photographie qui piège le voyeur dans la logique de son fantasme. L'image déréalise la souveraineté du regard dans la mesure où le voyeur photographe « receives the same look he gives ${ }^{10} »$. Il expose ainsi son plaisir au regard, au risque de perdre en retour la mise de son désir dans le champ visuel. À l'image de Newton, Burgin se met à son tour en scène dans l'espace pervers de la photographie. Il prend dès lors la place du scopophile coupable de regard, dans un jeu de déplacement du point de vue. Ainsi le voyeur doit-il s'acquitter par la parole de la fixation de l'image, afin de prolonger la vision. Il devise devant l'image, il parle, il nous entretient de l'image pour payer sa dette de vision et de plaisir ${ }^{11}$. Il parle et ainsi se lève l'idée de sainte Sébastienne en lieu et place de la mise en scène d'une féminité.

Sébastienne est dans l'image comme son invisible. Elle se tient dans l'intervalle de ce que des images - l'autoportrait de Newton et Las Meninas de Vélasquez - disent du visible et donnent à en lire. Sous ce rapport, ce n'est sans doute pas un hasard si la sainte apparaît dans un miroir sur la même pellicule trouble qui piège le regard du souverain dans la composition de Vélasquez comme personnage surnuméraire. Ce n'est peut-être pas non plus un hasard si ce même miroir pervers parvient à piéger John Searle lorsqu'il l'identifie au point depuis lequel le tableau doit être regardé. Le centre géométrique de la composition perspectiviste se situe toutefois ailleurs, sur la main du personnage qui se tient dans l'encadrement de la porte ouverte à l'arrière de la scène. L'analyse optique de l'ensemble n'en demeure pas moins problématique comme le rappelle Hubert Damisch dans la dernière section de Lorigine de la perspective ${ }^{12}$. Damisch donne sens à cet effet de leurre en opérant une distinction entre le centre géométrique de la perspective et son centre imaginaire situé sur la même ligne horizontale que le point de fuite avec lequel le miroir entre en concurrence. Le corps analogique de Sébastienne convoque ainsi dans la composition de Newton le paradigme perspectiviste comme ordre d'un imaginaire du visible. L'image et

10. Ibid., p. 62.

11. Ibid., p. 63: "Caught looking, I (male spectator) must now suspect that I am only talking about this picture at such length in order to be allowed to continue looking. »

12. Hubert Damisch, L'origine de la perspective, Paris, Flammarion, coll. «Champs», 1987, p. 393. 
la femme, le modèle et la femme, la sainte au miroir et June hors reflet, hors le cadre du miroir, encadrent le lieu d'où le voyeur-photographe devrait être absent de son fantasme: «According to the conventions of the genre we would expect to see only the model [...]. But the isolating function of the framing edge has failed here, and it is precisely this function that a fetishistic relation to the image would demand ${ }^{13}$. » À la place de l'absence, il faut penser ce que Burgin identifie comme l'invisible de l'image, comme l'inscription - visuelle plus que visible, parce que d'abord de l'ordre de la virtualité de l'image - de la rencontre de Newton avec l'énigme visuelle de la théorie freudienne de la sexualité: «It is the trace of the encounter with the enigma of sexuality that is inscribed in Newton's picture. Reference to fetishism alone cannot explain why this picture looks the way it does ${ }^{14}$.» Tout se passe comme si, à la place de cette absence attendue, Sébastienne entrait en scène comme la sphinge de cette même énigme du visuel: «But what the man behind the camera will never know is what her sexuality means to her, although a lifetime may be devoted to the inquiry ${ }^{15}$. » Dans l'écart entre l'image - l'apparition de Sébastienne - et ce dont elle est en quelque sorte la faillite, devant l'impossibilité (devant l'image de cette impossibilité) de distinguer clairement (c'est-à-dire «naturellement», voire géométriquement) une sexuation de l'objet du désir, un autre regard sur la chose se fait jour.

Lespace pervers de la photographie de Newton rejoue la parade théorique «brunelleschienne» sur une autre scène qui aligne et encadre le reflet de l'œil du dispositif photographique entre le regard de June, à gauche, et le nombril du modèle sans nom, à droite. L'expérience optique conduite par Filippo Brunelleschi (1377-1446) est décrite par Antonio Manetti dans sa Vita di Brunelleschi ${ }^{16}$. Une peinture (représentation du baptistère San Giovanni dans l'expérience originale) est placée devant un miroir tenu à bout de bras. Un orifice de forme conique est creusé au centre du panneau peint derrière lequel vient se placer l'œil du spectateur. Le regard détaché de la présence visible du corps qui le motive est ainsi réfléchi dans le tableau tout en restant caché dans ses motifs. À la fois masqué et exhibé par l'appareil photographique, valant alors comme une prothèse exubérante, l'œil de Newton est mis entre parenthèses par deux regards féminins, ou plus précisément, est inscrit entre le focal de sa femme et celui du modèle... mais sous le regard de son nombril.

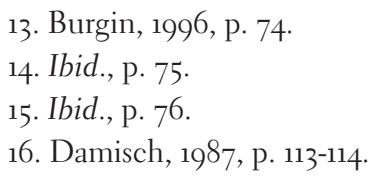


Au miroir du dispositif «brunelleschien », la transfiguration de la pin-up en figure de sainteté nous confronte à cet objectif ombilical, ombilic d'une image qui, en termes lacaniens, suspend l'élision du regard: «[É]lision de ceci que, non seulement ça regarde, mais ça montre ${ }^{17}$. Derrière le sex-appeal de Sébastienne, la «Grazia» de Sébastien pénétré de flèches se fait jour. L'image est en mouvement. Ou plutôt, elle est mouvement théorique. Ce battement de l'œil et du regard tantôt élidé par le voyeur, tantôt visualisé par l'hagiographe (sous la forme de l'apparition de Sébastienne) est matière à récits (si le mouvement est ici compris comme la forme d'une narrativité), voire matière du récit même où se négocie le lien entre le corps réel (celui du spectateur voyeur) et celui du saint (fiction du corps de sainteté et de ses propriétés apotropaïque ou cathartique). Daniel Arasse isole un moment de cette parade du regard et de son objet - entrée, retrait, esquive, rapprochement - dans une lecture d'un détail du Saint Sébastien d'Antonello de Messine, d'un détail qui nous confronte également à l'ombilic de l'image ${ }^{18}$. Quelque chose fait tache dans la représentation du martyr, quelque chose de l'ordre de la représentation et du corps, de la représentation du corps telle qu'elle s'intègre «intimement», ainsi que le souligne Arasse, dans un ensemble architectural «rendu» par une rhétorique de la perspective linéaire multipliant les indices angulaires (dallages, corniches, multiplication des ouvertures, contrastes...). Ce rapport intime d'inscription est cependant perturbé par la présence au pied du saint supplicié de quelque chose comme le débris d'une colonnade. Le reste d'une catastrophe dont nous ne conservons que ce fragment comme la promesse d'un autre récit témoignant de ce désordre.

Le nombril du saint n'est pas là où il devrait être. Il est déplacé, tout comme l'est le photographe dans l'autoportrait de Newton, qui lui non plus ne devrait pas être visible. Dans le tableau d'Antonello de Messine, l'ombilic est là, mais dévié de l'axe central du corps. Au regard de la fiction classique du corps architectural, il est déplacé. Le nombril - le particolare qu'une lecture iconologique reconnaît comme tel et identifie dans l'anthropomorphie vitruvienne - fait tache. Corrélativement, le déplacement latéral du nombril de Sébastien par rapport à l'axe vertical du point de fuite de la composition, lieu de projection géométrique de l'œil du spectateur, se présente à Daniel Arasse comme une perversion de

17. Jacques Lacan, Le séminaire XI. Les quatre concepts fondamentaux de la psychanalyse, Paris, Éditions du Seuil, 1973, p. 72.

18. Daniel Arasse, «Le corps fictif de Sébastien et le coup d'œil d'Antonello », dans Ivàn Almeida et Claude Reichler (dir.), Le corps et ses fictions, Paris, Les Éditions de Minuit, coll. «Arguments», 1983, p. 55-72. Pour le tableau en question, voir Antonello de Messine, Saint Sébastien, Dresde, Gemäldegallerie, 1476. 
la scénographie théorique «brunelleschienne» visant à mettre en évidence la coïncidence entre le point de fuite et le point de vue (ou point du sujet) dans la géométrie perspectiviste. À supposer que le Sébastien d'Antonello actualise - au sens linguistique - ce dispositif, il en résulte que le point géométrique du sujet et son lieu d'inscription symbolique se voient différés dans l'espace de la représentation, autorisant de ce fait la reconfiguration de l'umbiculus en oculus de la peinture même, dans une conversion iconique de la tache fixant/visant le voyeur. Arasse rappelle toutefois dans une note de bas de page que l'apparition troublante de cet éclat dans l'ombre du nombril - «[...] clairement identifiable jusque dans le léger accent lumineux qui éclaire la pupille ${ }^{19} \ldots »$ - résulte d'une transgression des conditions d'exposition de la peinture, ainsi que de la scénographie de sa contemplation. Ce détail renvoie à l'intimité du voyeur avec la peinture, devant elle comme au plus près d'elle, à même le pigment. De même, l'objectif de ce rapprochement de l'œil de l'historien qui d'une certaine manière problématise la distance qui pose l'objet ne consiste pas à mettre en lumière un inconscient du peintre ou de la peinture. Si le détail a valeur de symptôme, c'est au sens critique (et non pas clinique) que lui donne Georges Didi-Huberman. Il est le phénomèneindice d'une relation anthropologique instable à l'image ${ }^{20}$. Le symptôme assure le passage du corps à l'image et identifie la relation de discontinuité qui caractérise une sexuation du visible: «Un symptôme [...] ce sera par exemple le moment, l'imprévisible et immédiat passage d'un corps à l'aberration d'une crise, d'une convulsion hystérique ${ }^{21}$.» Dans l'autoportrait de Newton, l'alignement de l'objectif du photographe, du nombril du modèle, et du regard de June est symptomatique d'une instabilité des assignations, qui donne sens à la venue de Sébastienne dans l'image: «In the space of events in which this vignette is situated nothing is fixed, everything is mobile, there is no particular aim ${ }^{22}$.»

Cette intervention par le détail, et ce qu'elle pose d'une certaine intimité avec la peinture, problématise la corrélation entre la systématisation de la représentation perspectiviste et l'émergence du corps de Sébastien en peinture, émergence de sa nudité auparavant recouverte d'un vêtement ou des flèches de son supplice, à dater de la seconde moitié du $15^{\mathrm{e}}$ siècle. Cette corrélation rend possible l'apparition du corps de Sébastien comme «fiction du corps désiré » dans une scénographie

19. Arasse, 1983, p. 68

20. Georges Didi-Huberman, Devant l'image. Question posée aux fins de l'histoire de l'art, Paris, Les Éditions de Minuit, coll. «Critique», 1990, p. 114.

21. Ibid., p. 306.

22. Burgin, 1996, p. 75. 
complexe. Celle-ci le représente face à ses archers qui ont déjà exécuté leur œuvre (cinq flèches ont atteint leur cible) et qui le re-présentent devant les yeux des fidèles. Dans un face-à-face avec le corps du saint, piégés dans l'espace pervers de la peinture, ils se retrouvent ainsi dans la même position que les archers dont il leur faut pourtant se distinguer. L'image oscille entre le regard qui blesse et celui qui adore. Paradoxalement, le regard du saint martyr échappe à cette dioptrique. Prenant le ciel à témoin, il est ravi au cône de la vision et du supplice. Nous nous retrouvons donc confrontés à cette autre transaction entre le regard sur la peinture et ce que Daniel Arasse appelle l'œil de la peinture, ce troisième œil qui renvoie aux archers leur regard meurtrier. Dans la syntaxe perspectiviste, telle que l'explicite Damisch en reconstituant les implications théoriques de l'expérience de Brunelleschi, le décalage de l'umbiculus et de l'oculus acquiert une valeur énonciative. Corrélativement, dans la parade théorique d'Antonello et d'Arasse s'énonce quelque chose de l'ordre de la loi du visible qui lie indissociablement le supplice au visuel. Le délié qui apparaît comme tel, symptomatiquement, dans la description du détail, renvoie dès lors à la nature de ce lien entre le supplice et le regard comme à l'énigme du partage du regard. La relation entre les corps réels et fictifs de la peinture que la «fiction du corps désiré» de Sébastien organise, est d'abord apotropaïque. Le saint est réputé protéger la cité des fidèles des assauts morbides de la peste noire. Cette fiction repose sur une identification-projection:

En tant que le dévot est membre de la communauté chrétienne, il est, en son corps collectif, protégé par le corps particulier du saint coreligionnaire; mais, en tant que corps lui-même particulier, le dévot se met à l'abri du corps singulier de Sébastien. Il existe ainsi une certaine hésitation dans la relation possible entre les deux corps du dévot et du saint; incertitude qui renvoie à un phénomène fondamental qui se joue alors et que j'appellerai - sans vouloir ici entrer dans le détail de l'analyse - le "processus d'individuation» du corps propre dans la culture et les pratiques du temps ${ }^{23}$.

Par la suite, au cours du $15^{\mathrm{e}}$ siècle, rappelle Arasse, Sébastien se dote d'une autre propriété, cette fois-ci cathartique. Il apaise les voluptueux et les concupiscents. D'une fiction du corps à l'autre, d'une vertu à l'autre, on peut toujours rêver aux évidences (comme aux images et apparitions) d'une corrélation entre ce procès de stigmatisation/culpabilisation (d'une culpabilité dans laquelle un partage du regard est en jeu) et l'histoire de l'individuation du corps. Confronté à son incarnation chez Antonello de Messine, le voyeur - le photographe dans l'autoportrait de Newton - est traduit devant le même tribunal, appelé devant l'image. 
L'apparition de Sébastienne provoque le voyeur, comme dans La légende dorée Sébastien provoque ses bourreaux ${ }^{24}$. Elle vient ainsi déplacer le lieu dont s'autorise le discours du théoricien et la constitution du paradigme même de la théorie dans son rapport à la position (socioculturelle, symbolique, institutionnelle, sexuelle et gendered) que son exercice lui permet d'occuper en tant que sujet souverain et sujet du discours de savoir dans la modernité occidentale. Sébastienne réapparaît dans une gravure de Louise Bourgeoi ${ }^{25}$. Il n'est plus question de jeux de miroirs et de perspectives complexes. Le corps de la sainte y est au contraire mis à plat ${ }^{26}$. La martyre, décapitée conformément au Martyrologe romain $^{27}$, est représentée sous les traits d'une plantureuse créature interdite de regard. La plaie du cou évoque, par sa texture composée de traits concentriques, ceux d'une cible. Les flèches qui semblent venues tout droit de l'iconographie du martyre de Sébastien (ou alors venues d'autres extases baroques réservées aux saintes mystiques) l'assaillent de toutes parts et l'épinglent comme un insecte énigmatique, renvoyant à cet autre voyeurisme propre à l'œil de l'anatomopathologiste qui s'évertue à rendre visible ce que le corps occulte. En effet, ces flèches sans archers apparents (seront-elles pour autant objectives?) - l'extrémité de l'une d'elles est explicitement réservée au nombril de la sainte - détaillent le corps représenté comme sur une planche anatomique pour composer un inquiétant chevalet du visible, du lisible et du corps hystérique mis en scène ${ }^{28}$. Cette Sébastienne de Louise Bourgeois qui semble danser dans son supplice suspend sa «légende »: à la fois ce que l'on peut et ce qu'il faut lire d'elle suivant le fléchage de son corps tout en striures. Son corps hermétique suspend toute indexation de son image. Cette estampe de 1992 était visible lors de l'exposition Hysteria and the Body (2008) organisée par le Musée des Beaux-arts du Canada. Dans ces conditions d'exposition, le martyre de la sainte acéphale vient déjouer les mascarades fétichistes et les effets de théâtralisation de l'invisible féminin associés aux

24. Jacques de Voragine, La légende dorée, tome I., trad. Jean-Baptiste Marie Roz, Paris, Garnier Flammarion, 1967, p. 139.

25. Louise Bourgeois, Sainte Sébastienne, 1992. Pointe sèche sur papier vélin, imprimé par Harlan \& Weaver, intaglio 120,5 x 94,3 cm, image 98,7 x 78,3 cm. Reproduction en ligne: www.gallery.ca/en/see/collections/artwork.php?mkey=43071 (dernière consultation le 16 juin 2011).

26. Mulvey, 1975, p. 12.

27. Jean Baptiste Carnandet, Le martyrologe romain, Lyon, Gauthier, 1866, p. 245.

28. Georges Didi-Huberman, Invention de l'hystérie. Charcot et l'iconographie photographique de la Salpétrière, Paris, Macula, 1982. 
leçons du regard prononcées par Charcot ${ }^{29}$. L'œil de la gravure - sur le modèle de l'œil de la peinture chez Antonello de Messine - se situe quelque part là, entre le corps fictif et le corps réel de la martyre, là où la plaie du cou se fait tantôt focale, tantôt cible.

\section{III.}

Le retour de Sébastienne dans la photographie de Newton sous les traits de son punctum donne corps à une différence qui convertit en narrativité le temps consacré à cette reconstitution, le temps passé sur les détails de l'image, de même que l'attention et l'étude accordées à leur étrangeté. Au terme de ce temps d'étude (studium), alors que se relâche l'attention et au moment indéterminé où il faut en finir avec les projections pour en venir au réel, l'apparition perd de son étrangeté en échangeant son incongruité contre un discours de savoir sur sa réalité historique (en particulier dans le domaine de l'histoire de l'art). Par ailleurs, une sorte de conversion s'effectue dans la mesure où le détournement du regard de sainte Sébastienne nous renvoie à la logique de l'opération historiographique qui situe au sein d'un même espace de langage, l'absence et la logique de production qui en dépendent ${ }^{30}$.

Pour Burgin, la césure photographique du temps qui se révèle dans le punctum nommé «Sébastienne» autorise un présent organisé autour de l'énigme d'une présence ${ }^{31}$.

Sooner or later, as in Newton's image, we open our eyes, come back to a tangible reality: here that of the woman's body. That which is physical, that which reflects light - which has here left its trace on the photosensible emulsion. But what the man behind the camera will never know is what her sexuality means to her, although a lifetime may be devoted to the inquiry ${ }^{32}$.

Tôt ou tard («sooner or later»), une fois l'éclat du flash dissipé, entre deux clignements du troisième œil, commence un récit. Cet autre récit, cet autre discours sur l'image qui n'est pas discours de savoir, qui ne relève pas d'un savoir

29. Voir Kaitlyn Patience, «Hysterical Spaces, Curatorship and Meaning in the Traveling Exhibition: A Case Study of the National Gallery of Canada's "Hysteria and the Body" ", Shift. Queen's Journal of Visual \& Material Culture, vol. 3, 2010, p. 1-23. En ligne: www.shiftjournal.org/articles/2010/Patience.pdf (dernière consultation le 16 juin 2011).

30. Michel de Certeau, L'écriture de l'histoire, Paris, Gallimard, coll. «Folio Histoire», 1975, p. 12.

31. Barthes, 1980, p. 120-121.

32. Burgin, 1996, p. 76. 
historique, ni de la forme historiciste du savoir et de son récit, se détache comme une fable sur fond de l'histoire freudienne de la découverte de l'invisible et de sa prise de possession théorique telle que la reconstitue par exemple Jane Gallop: «Freud articulated the "discovery of castration" around a sight: sight of a phallic presence in the boy sight of a phallic absence in the girl, ultimately sight of a phallic absence in the mother. Sexual difference takes its significance from a sighting ${ }^{33}$. » À travers le grand miroir du studio, l'image de Sébastienne investit le visible et le discours du visible (ce qu'un discours de savoir freudien investit dans le visible) en renvoyant le corps du voyeur occulté par l'écran du dispositif «brunelleschien » à la mascarade des objectivations de son désir. Un autre regard se fait jour qui soustrait l'image à son objectivation et à sa stabilité en la dotant d'un droit de regard qui de ce fait mobilise le spectateur. Il le renvoie à son voyeurisme, à sa relation au lieu vide et à la gravité de ce qu'il ne voit pas et qui de toute façon ne saurait être visible dans le tombeau fétichiste du réel: «N'est-ce donc que cela, ce trou de chair, à quoi mon désir renvoyait? Qu'en est-il donc de mon propre désir ${ }^{34}$ ?» Sur le seuil que trace l'apparition et réapparition de la figure de sainteté devant la photographie de Newton, la vision est renvoyée au désir de voir qui lui a donné lieu ou au désir d'occulter ce qu'il ne saurait voir. À l'intersection de ces fléchages de l'œil et du regard (Burgin répondant à Laura Mulvey répondant indirectement au voyeurisme de Newton, Arasse répondant au Sébastien d'Antonello de Messine répondant à La légende dorée, Damisch répondant à Brunelleschi répondant à Alberti, Didi-Huberman répondant à Vasari et Panofsky), l'absence et la peur devant l'absence que les mécanismes de fétichisation incarnent dans le champ de la vision (elle n'a pas... elle n'est pas/elle n'est que...) comme marqueur de la différence sexuelle se retrouvent projetées dans, ou sur le vide au cœur de l'idéologie de la représentation telle que la modélise la perspective "brunelleschienne », et telle que Foucault cherche à l'énoncer dans sa lecture des Suivantes. Il n'y a pas ici d'échange historiographique, d'échange du visible contre du lisible, mais bien inscription d'une différence hagiographique.

«À l'extrémité de l'historiographie, comme sa tentation et sa trahison, il existe un autre discours. On peut le caractériser [...] comme le corpus d'une différence ${ }^{35}$.» Cette tentation est à entendre dans le sens d'un travail du virtuel,

33. Jane Gallop, The Daughter's Seduction: Feminism and Psychoanalysis, Ithaca, Cornell University Press, 1982, p. 27.

34. Michel Montrelay, «Recherches sur la féminité », Critique, n² 278, 1970, p. 666. 35. De Certeau, 1975, p. 274. 
à la fois du désir dans l'image (du désir de se faire image, ou à l'image de) et comme un possible du discours du savoir sur l'image que l'on retrouve dans ces trois questions corrélées: À partir de quand le désir devient-il socialement acceptable et au delà de quoi (de quelles limites) devient-il inacceptable? À partir de quel seuil du visible le désir est-il acceptable ou, à l'inverse, inacceptable? À partir de quelle(s) image(s) le désir de l'autre est-il identifié au désir visuellement inacceptable? Avant de pouvoir prétendre répondre à cette triangulation, il faudra d'abord se demander quel genre de réponse on est en droit d'attendre et de donner, et dans quel récit il faudra s'engager. L'hagiographie est du côté de la fable que traduit, ordonne, réorganise et à la limite rejette une pratique discursive afin de se constituer en discours de savoir: «Du fait que la sélection érudite retient seulement des documents ce qu'ils ont de "sincère" ou de "véritable", l'hagiographie non critique (qui reste la plus importante) s'isole. Un clivage s'opère ${ }^{36}$. $\gg$ Ce qu'il y a de récit dans l'apparition de Sébastienne et dans le mouvement même de son apparition, dans le passage d'une image à une autre, participe de ce travail de la différence. En cela, son événementialité - la venue ou l'invention de l'autre, pour dérouler le fil conceptuel derridien dans Psyché est comparable à la visualité du blanc «illisible» des fresques de Fra Angelico à San Marco, qu'interroge Georges Didi-Huberman dans le premier chapitre de Devant l'image ${ }^{37}$. L'apparition de sainte Sébastienne comme événement intermédial se tient en marge du discours de l'histoire de l'art sur l'image aux côtés des ex-voto et des objets de dévotions (bòti) qui s'empilaient par exemple dans la basilique de la Sanctissima Annunziata à Florence ${ }^{38}$. Georges Didi-Huberman se penche sur l'existence et la disparition de ces autres images afin de répondre à la question qu'il pose aux fins d'une histoire de l'art: «[C]omment inclure, dans la méthode historique, cette efficacité - visuelle - du virtuel. Mais que pourra bien signifier la virtualité d'une image en histoire de l'art ${ }^{39}$ ? » La réponse se situe donc du côté de l'imagination hagiographique de Burgin, dans l'espace pervers de la photographie de Newton, là où la virtualité de sainte Sébastienne donne sens à la réciprocité d'une opération critique visant à altérer l'image du désir et à qualifier la désirabilité de cette altération.

36. Ibid., p. 276.

37. Didi-Huberman, 1990, p. 21-36.

38. Ibid., p. 262-264.

39. Ibid., p. 41. 